\title{
85 Other viral infections
}

\author{
Stanley A. Gall
}

\section{HUMAN PAPILLOMA VIRUS}

Human papillomavirus (HPV) is an icosahedral, nonenveloped, double-stranded deoxyribonucleic acid (DNA) virus of approximately $55 \mathrm{~nm}$ in diameter (1). The genome of HPV s consists of approximately 8 kilobase paired molecules of circular, double-stranded DNA. All the proteins are encoded on one of the two DNA strands. All papilloma viruses share a similar genomic organization consisting of an early (E) gene region, a late (L) gene region, and a regulatory region. The five "early" proteins (E1, E2, E5, E6, E7) are required for viral replications and/or cellular transformation. Transformation of the "late" structural L1 and L2 transcripts and of the E1 to E4 spliced transcript is restricted to differentiating epithelium where viral assembly occurs $(2,3)$. The L1 protein comprises the majority of the virus shell. The L1 gene is the most highly conserved gene among individual types. The $\mathrm{L} 2$ protein of $77 \mathrm{kDa}$ is known as the minor capsid protein because it contributes a smaller percentage of the capsid mass than does the L1 protein.

More than 100 different types of HPV have been identified and fully sequenced and at least an additional 50 to 100 putative types have been partially characterized (4). HPV types are assigned numbers/designations when the DNA sequence has been established and a comparison with previously known types has found less than $90 \%$ sequence identity in the L1, E6, and E7 genes of the virus (5). An isolate with more than $90 \%$ homology to a known HPV type is classified as a subtype.

All HPV types are epitheliotropic and fully differentiated squamous epithelium is required for completion of the HPV life cycle. HPV infects keratinocytes, the predominate cells of epithelial surfaces. It is believed that only basal or undifferentiated keratinocytes are infected. The viral replication cycle is complicated as the keratinocytes undergoes the process of differentiation. Virus is assembled in the nuclei of most differentiated keratinocytes and can be detected in these cells as desquamation occurs from the epithelium (6).

HPV is transmitted by close contact with desquamated keratinocytes from an infected individual. The desquamated keratinocytes contain viral particles that are thought to infect a new host through microabrasions in the epithelial surface (7).

Approximately $40 \mathrm{HPV}$ types infect the genital tract. HPV infection of the human genitalia causes a range of clinical states including asymptomatic infections, genital warts, abnormal pap smears, preinvasive and invasive cervix, and vaginal and vulval cancers (8). The different HPV types can be subdivided into two categories: "high risk and "low risk" based on whether the HPV could be found in cancer specimens. The "low-risk" types such as HPV-6 and HPV-11 are associated with benign hyper-proliferative lesions commonly referred to as genital warts or condyloma acuminata and cause abnormal pap smears and low-grade dysplastic lesions (9). "High-risk" HPV types such as HPV-16 and HPV-18 cause low- and high-grade dysplastic lesions of the cervix and invasive cervical cancer. Other HPV-related genital malignancies include vulval cancer (10), vaginal cancer, and anal cancer (11). Approximately $45 \%$ of penile cancer is associated with HPV (12). HPV-16 causes more than $50 \%$ of the non-cervical genital tract cancers. The HPV types most commonly associated with anogenital lesions are HPV-6, -11, -16, -18, -31, -33, -35, -45, -52, and -58 (13).

\section{Epidemiology}

Genital HPV infection is primarily transmitted through sexual intercourse. In, virtually, all studies of HPV prevalence and incidence, the constant predicators of infection have been measures of sexual activity, most importantly the number of sex partners (lifetime and recent) $(14,15)$. Transmission of HPV through other types of genital contact in the absence of penetrative intercourse (i.e., oral genital, manual genital, genital-genital contact) has been described but is less common than that through sexual intercourse. Genital HPV transmission by nonsexual routes is uncommon. Nonsexual routes include transmission from a mother to a newborn baby (16).

The 2002 National Survey of Family Growth indicated that $24 \%$ of females in the United States were sexually active by age 15 years (17) increasing to $40 \%$ by age 16 years and $60 \%$ by age 18 years.

\section{Natural History of HPV Infection}

The majority of HPV infections are transient and asymptomatic and cause no clinical problems. The majority (70\%) of new HPV infections clear within 1 year and approximately $90 \%$ clear within 2 years $(15,18)$. The median duration of new infections is 8 months. Persistent infection with the same highrisk oncogenic HPV is a prerequisite for cervical cancer precursors and invasive cervical cancer (19).

\section{HPV Prevalence and Incidence in the United States}

It is estimated that 6.2 million new HPV infections occur every year among persons aged 14 to 44 years $(20,21)$.

This is an underestimate as HPV infections occur in persons younger than age 14 and over 44 years $(22,23)$. Modeling estimates suggest that $>80 \%$ of sexually active women will have acquired genital HPV by age 50 (24).

HPV infections are not reportable diseases. The HPV disease spectrum includes abnormal cytology, genital warts, low-grade squamous intraepithelial lesion (LGSIL), -highgrade squamous intraepithelial lesion (HGSIL), carcinoma in situ, adenocarcinoma, and invasive squamous cell carcinoma (Table 1). Evaluations from gynecologic exams from a variety of offices and clinics have documented the prevalence of HPV DNA ranging from $14 \%$ to $90 \%$ (25). Prevalence was the highest among sexually active females aged $<25$ years and 
Table 1 Incidence of Human Papillomavirus Spectrum Diseases Annually in the United States

$\begin{array}{lc}\text { Cervical cancer (21) } & 11,000 \\ \text { HGSIL (22) } & 330,000 \\ \text { LGSIL (22) } & 1,400,000 \\ \text { Genital warts (23) } & 1,000,000 \\ \text { Abnormal cytology } & 1.5-2.0 \text { million } \\ \text { Abbreviations: HGSIL, high-grade squamous intraepithelial lesion; LGSIL, } \\ \text { low-grade squamous intraepithelial lesion. }\end{array}$

decreasing with age (26). In a study of sexually active women aged 18 to 25 years, the prevalence of any HPV was $26.9 \%$. Prevalence of types 6 or 11 was $2.2 \%$ and prevalence of types 16 or 18 was $7.8 \%$ (27). Prevalence was the highest among women aged 20 to 24 years $(44.8 \%)$.

Studies of incident HPV infections that have evaluated HPV DNA detection over time demonstrate that acquisition occurs soon after sexual debut. In a prospective study of college women in the United States, the cumulative probability of incident infection was $38.9 \%$ by 24 months after first sexual intercourse. HPV-6 acquisition was highest (10.4\%); 5.6\% had acquired HPV-18 (14).

\section{Clinical Sequelae of HPV Infection}

In the United States, approximately 50 million women undergo annual pap cytologic testing; approximately 3.5 to 5.0 million of these tests will require follow-up including 2.0 to 30 million with atypical squamous cells of undetermined significance (ASC-US), 1.25 million LGSIL, 300,000 HGSIL, and 1.0 million with genital warts (28).

All anogenital warts (condylomata) are caused by HPV and approximately 90\% are associated with HPV types 6 and 11 . The average time for the development of new anogenital warts after infection with HPV types 6 and 11 is 2 to 3 months. Not all persons who acquire HPV types 6 and 11 develop warts. Approximately $30 \%$ of warts regress spontaneously except during pregnancy or in states of immunosuppression when the rate of regression is much lower.

Reoccurrence of anogenital warts tends to be more common following periods of immunosuppression, either exogenous or endogenous. Infection with HPV resulting in clinical genital warts will lead to activation of cell-mediated immunity, which will prevent infections with the same HPV type. However, since many HPV infections are not cleared but become latent, immunosuppression will be followed by either active genital warts or abnormal cytology. In a majority of women who develop genital warts during pregnancy, by 6 weeks postpartum, the genital warts are totally resolved or dramatically decreased in size.

Infection in the tracheobronchial tree with HPV types 6 ad 11 may result in recurrent respiratory papillomatosis (RRP), a disease characterized by recurrent warts or papillomas in the upper respiratory tract, particularly the larynx. RRP is divided into juvenile onset (JORRP) and adult onset based on diagnosis before or after 18. JORRP is believed to result from vertical transmission of HPV from mother to infant during delivery, although the median age of diagnosis is 4 years. A multicenter registry of JORRP during 1999 to 2003 demonstrated that although the clinical course of JORRP was variable, it is associated with extensive morbidity, requiring a median of
13 lifetime surgeries to remove warts and maintain an open airway. Estimates of the incidence of JORRP range from 0.12 to 2.1 cases per 100,000 children aged $<18$ years in two cities $(29,30)$.

\section{Prevention of HPV Infection}

Condom use may reduce the risk for HPV and HPV spectrum diseases (e.g., genital warts and cervical cancer). Pap testing can detect precancerous changes in the cervix by evaluation of cytology, and HPV vaccines can prevent HPV diseases due to HPV types in the HPV vaccines.

At the current time, two HPV vaccines are licensed by FDA. The vaccine licensed in June 2006 was the quadrivalent HPV vaccine (HPV types $6,11,16,18$ ) Gardasil ${ }^{\circledR}$ manufactured by Merck \& Co. The second HPV vaccine, a bivalent vaccine (HPV types 16,18) Cervarix ${ }^{\circledR}$ licensed by the FDA in October 2009, was manufactured by GlaxoSmith Kline. The quadrivalent vaccine is licensed for women for the prevention of HPV types 6, 11, 16, 18 related cervical, vaginal, and vulval cancer precursor and dysplastic lesions and genital warts. In October 2009, the quadrivalent vaccine was licensed by the FDA for the prevention of genital warts on males of age 9 to 26 years. The bivalent vaccine, Cervarix, has been licensed for the prevention of cervical cancer precursors and cervical cancer due to HPV types 16 and 18 (Table 2).

\section{Recommendations for Vaccine Use}

Advisory committee on immunization practices (ACIP) recommends routine vaccination of males and females aged 11 to 12 years with three doses of quadrivalent HPV vaccine. The vaccine series can start as early as age 9 years. Vaccinations are also recommended for males and females aged 13 to 26 who have not previously been vaccinated or who have not completed the full series. The recommendation for the bivalent vaccine is similar but does not have the male indication.

At this time, neither HPV vaccines are recommended for use in pregnancy. However, pregnancy testing is not needed before vaccination. Any exposure to the vaccine during pregnancy should be reported to the appropriate vaccine registry:

1-800-986-8999 (Merck + Co) for quadrivalent
vaccine
$\begin{aligned} & \text { 1-888-452-9622 (GlaxoSmith Kline) for bivalent } \\ & \text { vaccine }\end{aligned}$

\section{MOLLUSCUM CONTAGIOSUM}

Molluscum Contagiosum is a human disease with a worldwide distribution and is found in persons of all age and caused by members of the Poxvirus group (31). The disease is spread by sexual intercourse or fomites.

The disease is characterized by a long incubation period from 2 weeks to 2 months. The disease presents by the appearance of firm, shiny, flesh-colored lesions that become pearly white and umbilicated and discharge a caseous material. The lesions are characterized by proliferation, hyperplasia, thickening, and degeneration of the epidermis. The infected cells become enlarged and develop large intracytoplasmic eosinophilic hyaline inclusion bodies. The lesions are not pruritic and they measure 1 to $5 \mathrm{~mm}$ in diameter but may form larger clusters. The lesions typical of molluscum contagiosum 
Table 2 Differences in Human Papillomavirus Vaccines Currently Licensed by FDA

\begin{tabular}{|c|c|c|}
\hline & Quadrivalent, Gardasil ${ }^{\circledR a}$ & Bivalent, Cervarix ${ }^{\circledR a}$ \\
\hline Licensed by FDA for women & June 2006 & October 2009 \\
\hline Licensed by FDA for males & October 2009 & None \\
\hline HPV types in vaccine & $6,11,16,18$ & 16,18 \\
\hline Age of vaccine recipients & 9-26 years & $9-26$ years \\
\hline Recommended by ACIP-women & June 2006 & October 2009 \\
\hline Vaccine administrators' schedule & $0,2,6$ months & $0,1,6$ months \\
\hline
\end{tabular}

are characterized by complete lack of immunocompetent cells in the epithelial component of the lesions (32).

The principal sites of involvement include the eyelids, face, trunk, and anogenital areas. Molluscum lesions evolve over 1 to 2 months and are frequently traumatized resulting in autoinoculation. Secondary bacterial infections may occur. The lesions may persist for as long as 1 year but have persisted longer. Spontaneous regression without scarring usually occurs and recurrences are rare. Patients who are immunosuppressed may develop extensive involvement (33).

The diagnosis of molluscum contagiosum is usually made by inspection of the characteristic umbilicated white papule. The differential diagnosis includes lichen planus, verrucous papules, basal cell epithelioma, and pyogenic granuloma. If the diagnosis is in doubt, the caseous material should be examined for intracytoplasmic eosinophilic inclusions. Treatment by sharp curettage or expression of porous material will facilitate healing without scarring.

\section{INFLUENZA}

See chapter on Maternal Immunization (Chapter 71).

\section{PARVOVIRUS}

\section{Epidemiology}

The human parvovirus B19 is a member of the DNA virus family Parvoviridae (34). This virus is one of the smallest known DNA viruses approximately 18 to $25 \mathrm{~nm}$ in size, about $5.5 \mathrm{~kb}$ in length, and with single-stranded DNA (34). The virus lacks an envelope, but does code for at least two major structural or capsid proteins and at least one nonstructural protein.

The human parvovirus B19 was first discovered by British investigators in 1975 while screening blood specimens for hepatitis B antigen. Subsequently, B19 was found to be associated with aplastic anemia crisis and erythema infectiosum or "fifth disease" $(34,35)$. This virus has also been associated with chronic bone marrow suppression, arthritis or arthropathy, fetal hydrops, and purpura.

Infection with parvovirus B19 is worldwide and is commonly seen in children aged 5 to 14 years. Outbreaks are most common in winter and spring, but there is a year-toyear variation in incidence. Estimates of positive seroprevalence rates in adults range from $28 \%$ to $72 \%(34,36)$.

Parvovirus is mildly contagious with an infection rate of $30 \%$ following primary exposure. The highest rates of secondary infection are seen in household exposure where the infection rate is as high as $50 \%$ (37).
Transmission of the virus is by respiratory droplets, and the incubation period is 4 to 14 days. Viremia develops 7 to 8 days after initial infection, lasting approximately 4 days (34). The rash associated with B19 appears following the viremic phase. The primary host is no longer infectious once the rash appears. Initial infection and seroconversion provide the host with lifelong immunity and reinfection is rare.

\section{Pathophysiology}

Parvovirus B19 viral replication and encapsulation take place in the host cells' nucleus. Host cell enzymes are used in viral replication. Rapidly dividing cells are preferentially infected, perhaps a product of $S$ phase of cell replication, a requirement for viral growth. Parvovirus B19 is particularly trophic and cytotoxic for erythroid progenitor cells $(34,35)$.

Following initial incubation and viremia, there is a transient inhibition of erythropoiesis, and reticulocytopenia occurs in the infected host. Similar changes are seen in other blood cell lines to a lesser degree. The bone marrow depression is first seen approximately 7 days after the initial infection and lasts for approximately 7 days. In the host with normal red cell survival or in a non-immunosuppressed state, this bone marrow depression is usually asymptomatic and self-limited. Aplastic crisis is seen particularly in patients with abnormally shortened red blood cell survival, in conditions such as sickle cell anemia, heredity spherocytosis, pyruvate kinase deficiency, thalassemia, and autoimmune hemolytic anemia. The immunocompromised host may develop bone marrow suppression of all cell lines secondary to an inability to clear the virus.

Transplacental fetal infection and subsequent fetal anemia may result in fetal hydrops and demise. The fetus is an ideal host for human parvovirus B19 because of an immature immune system and accelerated erythropoiesis with markedly shortened red cell life span $(34,35)$. It has also been proposed that the marked fetal anemia observed may lead to tissue anoxic and subsequent high output or hypoxic cardiac failure (37). While transplacental infection may lead to fetal anemia and hydrops, parvovirus B19 is not a common cause of congenital anomalies or neonatal disease. The period of peak fetal morbidity following maternal infection is approximately 4 to 6 weeks after maternal symptoms (37). The fetal loss rate following maternal infection has been estimated to be as low as $2.5 \%(38)$.

The rash associated with human parvovirus B19 infection occurs 17 to 21 days after infection and is probably secondary to antigen-antibody reaction (35). Arthritis or arthropathy, a 
probable postinfection phenomenon, may manifest with a purpuric rash-like illness, often associated with other signs of vasculitis. This rash may begin as a vesiculopapular eruption and is often distributed in the buttocks and lower extremities.

\section{Diagnosis and Management}

As with many viral infections, infection with human parvovirus B19 may be asymptomatic or carry symptoms of only a mild flu-like illness. The rash is more common in children than in adults but adults are more likely to develop arthritic complaints. The rash is characterized as a lacy rash especially on the face providing the "slapped cheek" appearance. A variable rash on the extremities may follow with a morbilliform, confluent, circinate, or annular appearance. This usually does not involve the trunk, palms, or soles and may be pruritic. The rash usually disappears within 1 to 2 weeks but may recur especially with stress, exercise, sunlight, or bathing $(34,35)$.

Cultured human parvovirus B19 requires bone marrow cells and therefore clinically difficult. Enzyme-linked immunoassay for B19 antigen and antibodies are available to aid in diagnosis. Diagnosis can be made through DNA hybridization techniques including DNA amplification via the polymerase chain reaction (37). Maternal IgM antibodies can be detected in 3 to 4 days after onset of clinical illness, whereas IgG antibodies are seen within 7 days. IgM antibodies persist for 3 to 4 months and IgG antibodies persist for years (36). Patients who demonstrate positive IgG with a negative IgM are considered immune and carry little risk from exposure to parvovirus B19. Those patients who have had an acute infection will have a positive IgM and are at significant risk for an adverse fetal outcome.

Fetal infection can be monitored by ultrasound for evidence of fetal hydrops, and percutaneous umbilical blood sampling can be used to diagnose fetal anemia. Demonstration of parvovirus B19 antigens or parvovirus-specific IgM antibody DNA will confirm the diagnosis.

Serial middle cerebral artery (MCA) blood flow analysis can detect early signs of fetal heart failure.

Infection is strongly suggested if eosinophilic inclusion and marginated chromatin are seen in infected fetal erythroid precursors (36).

If the initial ultrasound examination of the fetus is normal, including MCA measurements, the fetus should be followed with serial ultrasound examinations up to 12 weeks after maternal symptoms. Ultrasound-documented fetal hydrops resolves spontaneously in some cases of infection. While transplacental infection and hydrops fetalis have been successfully treated with percutaneous exchange transfusion, it has been observed that spontaneous resolution is frequent. Another observation that fetuses older than 20 weeks seem to have more spontaneous resolution may occur because of a more developed immune system (38).

Maternal serum alpha fetoprotein levels are elevated in association with intrauterine infection prior to the development of fetal hydrops as detected by ultrasound. The value of the test is limited by its nonspecific nature. Also the value of intravenous gammaglobulin for postexposure prevention of infection has not been fully evaluated. Vaccines or antiviral therapies specific for parvovirus B19 have not been developed.

\section{ENTEROVIRUSES \\ Etiology}

Human enteroviruses are members of the family of RNA Picornaviridae and are some of the most common human viruses (3). There are at least 72 serotypes of enteroviruses. The four recognized classes of enterovirus are poliovirus, coxsackie A virus, coxsackie B virus, and echovirus. To date, there are three polio subtypes, 23 coxsackie A serotypes, 6 coxsackie B serotypes, and more than 30 echovirus serotypes (39-41). Echovirus serotype 72 has been identified as hepatitis A virus. This chapter will focus on non-polio enteroviruses. Hepatitis A virus will be discussed in another chapter.

Viruses of the genus enterovirus are small $(27 \mathrm{~nm})$, singlestranded RNA with approximately 7500 bases. These viruses are icosahedral and lack an envelope. Enteroviruses have a nucleocapsid that is composed of 60 structural subunits $(39,40)$.

\section{Epidemiology}

The true incidences of disease due to enteroviruses are not known and those infections are not reportable. It is thought that between $50 \%$ and $80 \%$ of infections are asymptomatic. Infections occur throughout the year and are most prevalent from June to October in temperate climates $(39,40)$. Serotypes vary by region or year. The usual route of transmission is by either respiratory droplets or the gastrointestinal route.

Of all the infections, $80 \%$ to $90 \%$ are in children and adolescents 16 years old or younger (40). Adults account for approximately $8 \%$ of the non-polio enteroviral infections. Neonatal infections within the first month of life are common. In one study, $12.8 \%$ of newborns acquired enteroviral infection within 1 month of life and all infants recovered without difficulty (39). Women contracting enteroviral infection in late pregnancy are usually asymptomatic while those displaying symptoms are mild and nonspecific.

\section{Pathophysiology}

Diseases attributed to enteroviruses include aseptic meningitis, encephalitis, myocarditis, hepatitis, pneumonia, pleurodynia, and orchitis. Though often asymptomatic, infection may manifest with rashes, pharyngitis and conjunctivitis, parotitis, pericarditis, and pancreatitis. Rarely, the infection may be fulminant and fatal.

Following initial infection, viral replication occurs in the submucosal lymphoid tissue and within 1 to 3 days can be identified in regional lymph nodes (39) with subsequent viremia. The virus can be found in distant lymphoreticular tissue, that is, liver, spleen, and bone marrow (39). After replication in the lymphoreticular tissue, a more generalized infection with inflammation occurs, affecting multiple organs or tissues such as the central nervous system (CNS), striated muscle, and the heart. Animal studies have demonstrated a shorter incubation period and higher virus titers in pregnancy. Susceptibility to infection appears to increase with gestational age, but reverts rapidly to that of the nonpregnant state following delivery (39). Sites of primary infection in the gastrointestinal tract do not show evidence of this destructive process; therefore, diarrhea will not ensue in most patients. 
The host immune response to enteroviral infection includes the production of both humoral and mucosal antibodies as well as a cytotoxic T-lymphocyte cellular response (39).

Most perinatal infections occur secondary to transmission of the virus to the neonate during parturition or in the immediate postnatal period (41). Vertical transmission of enteroviruses is rare but has been documented $(39,41)$. As for infection of children and adults, the severity of disease in the neonate may range from mild to fatal, causing diseases of the CNS, liver, or heart (41). The neonate who has acquired an enteroviral infection by vertical transmission in the immediate peripartum period is thought to be at greater risk of serious disease when compared with the infant who has become infected following postnatal transmission. This serious infection is thought to result from a lack of maternal antibodies to the enteroviral infection.

Less is known about neonatal infection with coxsackie A virus. There are case reports of in utero fetal death with disseminated coxsackie A virus. It is rare for coxsackie A virus to be passed to the fetus (42). There appears to be no association between echovirus or coxsackie B virus infection and an increased spontaneous abortion rate. Stillbirth in late pregnancy increases with both maternal echovirus and coxsackie B viral infection. The association between maternal coxsackie B virus infection and a slightly greater risk of fetal urogenital defects and congenital heart defects is tenuous $(39,41)$. Coxsackie B virus, particularly serotypes B3 and B4, has been implicated in neonatal myocarditis (43).

\section{Diagnosis and Management}

The typical presenting symptoms of maternal enteroviral infection are those of an upper respiratory tract infection such as fever of a few days of duration with or without rash. The patient may have abdominal pain of such intensity that it may be mistaken for abruptio placentae or chorioamnionitis in the pregnant patient. Rarely, the patient presents with aseptic meningitis or myopericarditis. A presumptive diagnosis can be made by demonstrating virus-specific IgM antibodies or by documenting rising IgG titers in maternal serum. Definitive diagnosis requires culturing of the virus from either respiratory secretions or stool.

If maternal infection is established more than 5 to 7 days prior to delivery, the maternal immune response provides IgG antibody that crosses the placenta and protects the neonate against serious illness. Multiple studies have indicated that when the neonate has severe echovirus, or coxsackie B virus infection, approximately $60 \%$ of the pregnant patients will have been symptomatic during the perinatal period, usually within 1 week of delivery.

There is no specific treatment, effective vaccine or reliable immune globulin or specific vaccine for enteroviral infection. Vertical transmission at birth causes the most neonatal harm. Owing to the absence of passive immunization via maternal antibodies, it is suggested that delivery should be delayed for over 1 week after the onset of disease.

\section{MUMPS}

\section{Etiology}

The mumps virus is an RNA virus in the paramyxovirus family. The virus has a lipid membrane envelope with both the neuraminidase and the hemagglutinin. The envelope carries
"S" antigen; however, the antigen does not cross-react with other paramyxoviruses. The core antigen " $\mathrm{v}$ " is more specific to the mump virus (44).

\section{Epidemiology}

Mumps is found worldwide and the virus is spread by respiratory droplets or fomites (44). Epidemics may occur especially in urban settings or where susceptible individuals are in close quarters. The infected person is contagious from 6 days prior to and up to 9 days after the onset of disease (45). The virus may be isolated from the throat, saliva, and urine during the first 3 days of illness and may be found in the urine up to 2 weeks after it has disappeared from other sites. The virus may also be isolated from blood, breast milk, and the testis.

As in the pre-vaccine era, mumps is predominantly a disease of school age children of ages 5 to 14 years. A live virus vaccine was introduced in 1967 but uptake of the vaccine was scant until 1977 when less than 50\% of children had been immunized (46). The incidence of mumps in pregnancy is estimated to be between 0.8 and 10 cases per 10,000 pregnancies.

\section{Pathophysiology}

Mumps is usually a benign and self-limited disease. Following infection and incubation, viremia occurs. The disease is asymptomatic in $30 \%$ to $60 \%$ of patients. Most symptomatic patients experience moderate debilitation (46). Typical symptoms include fever, malaise, and swelling of one or both parotid glands. If the parotid glands are swollen, other salivary glands may also be involved. Viral replication occurs in multiple organs as the virus is disseminated. Pancreatitis, arthritis, and myocarditis are rare complications (45). Approximately $20 \%$ of postpubertal males will develop orchitis. Infection of the thyroid and breast may occur in the postpubertal females.

The virus may be cultured from cerebrospinal fluid in the asymptomatic patient and meningeal signs can be seen in 5\% to $25 \%$ of infections (46). The incidence of mumps encephalitis may be as high as 5 per 1000 reported cases. The cranial nerves may be involved and the most common residual CNS symptom is deafness.

Symptomatic reinfection has been known to occur (45). Although mumps infection may be fatal, the mortality rate is extremely low. Fatal cases of mumps are more common in adults with approximately one-half of mumps-associated deaths in patients 20 years.

The mumps virus can infect the placenta and infection can cause transplacental infection. One study reported a $27 \%$ spontaneous abortion rate when mumps occurred in the first trimester (46). Mumps have not been associated with prematurity.

\section{Diagnosis and Management}

The diagnosis of mumps may be made on clinical symptoms if present. The diagnosis can be confirmed by serologic studies demonstrating virus-specific IgM or using titer of virus-specific IgG. The virus can be easily cultured in African green monkey kidney cells, embryonated hen's eggs, and human cells (47).

In contrast to other viral infections such as influenza or varicella zoster, mumps does not demonstrate increased 
virulence or severity in the pregnant patient (44). Treatment is supportive.

Special precautions with delivery in isolation if possible has been suggested when the obstetric patient has overt mumps or if the patient has had proven or suspected onset of mumps within the 10 days preceding delivery (45). The same recommendations are suggested for the nonimmune patient exposed in the 3 weeks prior to labor. Postpartum care should be provided in isolation, though there is no need to separate the patient and her infant, and breast-feeding should be permitted. It is estimated that approximately one-half of newborns delivered to patients with overt mumps will acquire asymptomatic disease and be contagious up to 4 weeks of age. Nonimmune hospital personnel should not care for patients with mumps pointing out the need for all hospital personnel to be current with their measles-mumps-rubella vaccination.

The measles-mumps-rubella (MMR) vaccine is an effective live-attenuated viral vaccine that is part of the childhood vaccination schedule when the first dose of MMR is recommended at 12 to 15 months of age and a second dose at 4 to 6 years. Two doses of MMR vaccine are recommended for students attending colleges and one dose for health-care workers (47). In response to the resurgence of measles in 1989, this recommendation also had the effect of decreasing outbreak of mumps in high schools and college students. A study from the United Kingdom using MMR vaccine indicated vaccine effectiveness of $64 \%$ after one dose and $88 \%$ after two doses (48).

During the first 6 months of 2006, the United States reported a mumps outbreak of 2597 cases in 11 states (49). The outbreak highlighted the limitation of the 1998 recommendations and ACIP issued new recommendations (Table 3). The MMR vaccine is not indicated for use during pregnancy. Women should avoid pregnancy for 1 month following vaccination. Inadvertent immunization during pregnancy is not an indication for termination of pregnancy. Immune globulin has not been shown to be effective in the prevention of mumps and is not recommended $(45,46)$.

\section{MEASLES}

\section{Etiology}

Measles is an exanthematous disease caused by a member of the paramyxovirus family. This virus differs from other

Table 3 The 2006 Advisory Committee on Immunization Practices Recommendations on Mumps

Acceptable presumptive evidence of immunity

Documentation of adequate vaccination with two doses of live MMR vaccine for

Adults including health-care workers

Students at post-high school institution

Routine vaccination for health-care workers

Persons born after 1957: two doses of MMR vaccine

Persons born before 1957: one dose of MMR vaccine

For outbreak settings

Children aged 1-4 years and adults: consider a second dose of MMR vaccine

Health-care workers born before 1957: strongly consider two doses of MMR vaccine (50)

Abbreviation: MMR, measles-mumps-rubella. members of this family in that it does not demonstrate a neuraminidase.

Prior to the introduction of measles vaccination in 1963, epidemics of measles were seen every 2 to 3 years with 500,000 cases reported annually and approximately 400 to 500 deaths attributed to the complications of measles. In the 1980s, the reported incidence of measles fell to 1500 to 3000 cases per year (44). Measles is primarily a disease of children between ages 2 and 6 years. The incidence of measles in pregnancy is rare with estimates ranging from 6 to 40 cases per 100,000 pregnancies (51). Congenital measles are rare.

Prior to vaccination, measles were very contagious with an attack rate of approximately $90 \%$ in the nonimmune patient. Transmission is by respiratory droplets with the mucosa of the nose, oropharynx, or conjunctivas as the portal of entry. The usual incubation period is 10 to 14 days but may be as short as 7 days. The patient is infectious for 2 to 3 days prior to symptoms and 3 to 4 days after the appearance of the rash.

\section{Pathophysiology}

After the initial infection and incubation, viremia occurs and the patient demonstrates an influenza-like illness. There is usually a 3-day prodromal period with fever, inflammation of the mucosal membranes, cough, and coryza. Examination of the pharyngeal mucous membranes may reveal pathognomonic Koplik's spots. Subsequently, the patient develops a generalized maculopapular exanthema and malaise (52).

Complications of measles can be very serious. The most common complication is otitis media. Secondary bacterial pneumonia may follow measles viremia and is the most lethal complication. Encephalitis may seen at any age and occurs in 1 per 1000 cases. If encephalitis complicates measles, it usually appears within 3 to 7 days after the appearance of the rash and carries a mortality of approximately $10 \%$. The overall mortality rate of measles is estimated at less than $0.1 \%$ but is higher in children $<1$ year of age (53).

\section{Diagnosis and Management}

In an epidemic, the clinical signs and symptoms may allow a clinician to make a diagnosis. The differential diagnosis of measles includes a number of viral exanthematous diseases. The case definition includes fever $\left(>101^{\circ} \mathrm{F}\right)$ rash for more than 3 days and cough, coryza, or conjunctivitis. Hemagglutination inhibition (HI) serologic tests are the most widely used for diagnosis. A fourfold rise between acute serum (within 3 days of the rash) and convalescent serum up to 20 days later is diagnostic. The HI antibody persists for years and is useful to determine immune status. The presence of IgM HI-specific antibody will also diagnose a new case of measles. The IgM antibody will usually disappear 2 to 5 days after the appearance of the rash.

There is a higher complication and mortality rate in pregnant women. Pneumonia has been noted to be the most frequent fatal complication (44).

The virus infrequently crosses the placenta. Gestational measles has been linked to a higher incidence of premature labor. Maternal measles may lead to premature labor and delivery and therefore increase neonatal mortality. Pregnancies complicated by measles have not been firmly associated with congenital malformations or a higher rate of malformations (51). 
Newborns receive passive immunity from maternally derived IgG measles-specific antibody secondary to either maternal infection or immunization.

Nonimmune patients can be protected against measles following exposure if they are provided with passively acquired immunoglobulin within 3 days of exposure. The recommended dose of IgG is $0.2 \mathrm{ml} / \mathrm{kg}$ body weight intramuscularly $(51,52)$.

Measles can be prevented with administration of MMRattenuated live virus vaccine prior to pregnancy. In 1985, Gazala et al. reported the outcome of five pregnancies at 28 to 34 weeks of gestation complicated by a measles epidemic in Israel from December 1981 to May 1982. All five pregnancies were complicated by premature delivery with a mean duration between onset of illness and delivery of 3.5 days. The infants demonstrated low birth weight, and three of four live births had severe respiratory distress. The fifth infant was stillborn. Each live-born infant received IgG intramuscularly at a dose of $0.2 \mathrm{ml} / \mathrm{kg}$ on day 1 of life. None of the infants demonstrated any clinical signs or symptoms of measles.

\section{RUBELLA}

\section{Etiology}

The rubella virus is a member of the togavirus group and is a spherical, enveloped RNA virus, 50 to $100 \mathrm{~nm}$ in diameter (54).

\section{Epidemiology}

Rubella has worldwide distribution and prior to vaccine availability, pandemics occurred approximately every 20 years, with epidemics in 6- to 9-year cycles $(54,55)$. Rubella is endemic throughout the year but in temperature climates demonstrates a peak incidence from March to May. Prior to rubella vaccination, the primary disease occurred in children aged 5 to 9 years where as in the postvaccination era there is a major shift to persons older than age 15 (55).

Transmission of rubella is by aerosolized respiratory droplets. The usual incubation period is 16 to 18 days with a range of 14 to 21 days $(54,55)$. The infected individuals may shed virus for 1 week prior to and for approximately 1 week after the appearance of the typical rash. The virus may be isolated from respiratory secretions during this time, but the virus may be present in the urine and blood prior to the rash (55). A mild prodrome of malaise, low-grade fever, headache, and conjunctivitis may precede the rash by 1 to 5 days.

\section{Pathophysiology}

The initial infection occurs on the respiratory mucosa where the primary viral replication occurs (54). During incubation, for approximately 1 week prior to the onset of the rash, the virus can be isolated from leukocytes, conjunctiva, urine, and stool. The immune response of the host is both humoral and cell mediated. Viral-specific antibodies may be detected within 24 to 48 hours of the onset of the rash.

Infection with rubella is usually self-limited and mild. Postauricular, suboccipital, and posterior cervical lymphadenopathy are distinguishing features of the disease. The adenopathy will often herald the onset of the typical rash.

The most significant complication occurs secondary to transplacental transmission of the virus to the fetus during maternal viremia. In utero, infection may occur at any gestational age (31). The virus may infect the placenta and result in granulomatous changes and necrosis of chorionic villi. Fetal rubella infection may cause spontaneous abortion or stillbirth. Once established, fetal infection is chronic and persists well beyond birth (55). The rubella virus may be persistent in the most severely affected infants for up to 1 year. The exact pathologic mechanism of fetal infection and subsequent tissue damage is not well understood. Rubella is not cytolytic like measles and inflammation is not a prominent feature of infected cells. Infected cells do show reduced mitotic activity and there is a reduction in this total number of body cells.

The rate of congenital infection and the effect of transplacental infection appear to vary with gestational age at the time of infection. In a large prospective study of 1016 women with confirmed rubella infection, the rate of congenital infection was $81 \%$ in the first 12 weeks, $54 \%$ at 13 to 16 weeks, $36 \%$ at 17 to 22 weeks, $30 \%$ at 23 to 30 weeks, $60 \%$ at 31 to 36 weeks, and $100 \%$ from 37 weeks to delivery (55). No congenital anomalies were seen if infection occurred after 16 weeks. Another prospective study found no congenital anomalies if maternal infection occurred after 17 weeks of gestation $(56,57)$.

The congenital rubella syndrome (CRS) symptoms in decreasing frequency are hearing loss, mental retardation, cardiac malformations, and ocular defects $(56,57)$. The greatest risk to the fetus is when maternal infection occurs in the first trimester. Congenital anomalies of the heart, eye, and CNS have been seen only if maternal infection occurred between 3 and 12 weeks. Deafness is the most common anomaly occurring in 58\% of affected offspring and in $40 \%$ as a single defect (56). Patent ductus arteriosus was the most common cardiac defect and accounted for $79 \%$ of the cardiac anomalies seen $(56,57)$.

\section{Diagnosis and Management}

The symptomatic patient may experience a syndrome of malaise, low-grade fever, and tender and swollen lymphadenopathy of the suboccipital, postauricular, and posterior cervical lymph nodes prior to the onset of a rash. The rash of rubella initially appears over the face and posterior to the ears as a small, faint pink circular macular rash that eventually spreads to the neck, trunk, and extremities. The rash may also appear as a general blush in the face and may be described as petechial or purpuric. The rash generally disappears in 3 days.

Clinical history and examination are not reliable for making a diagnosis. Culture is slow and expensive and not recommended. The diagnosis of rubella is confirmed by serology. Rubella-specific IgM and IgG may be detected by a variety of techniques including HI, ELISA, compliment fixation, and radioimmunoassay. As with other diseases, the presence of IgM indicates acute illness as does a fourfold rise with IgG. IgM will disappear after 4 to 6 weeks.

All obstetric patients should be screened for rubella community on their first physician visit. If a patient suspects possible exposure and her immunity has not been established, serology should be obtained immediately and retested in 3 weeks.

The patient who shows a fourfold rise in titer of IgG antibody has a confirmed infection. If this occurs in the first 20 weeks of 
pregnancy, the patient should be counseled regarding implications for the fetus and should consider termination of pregnancy.

Vaccines for rubella were licensed in 1969 and are available with measles and mumps as MMR vaccine. ACIP recommends vaccination of all infants between 12 and 15 months and an additional dose for adolescents. All patients who are nonimmune on obstetric screening should receive MMR immediately postpartum. There is no risk to the postpartum patient or to the breast-feeding infant. Contraindication to MMR vaccine includes pregnancy, febrile illness, immunodeficient states, or receipt of immune serum globulin within preceding 3 months.

The risk to the fetus of inadvertent administration of MMR in pregnancy is not known. In a review, there were no defects compatible with CRS in 552 infants born to 635 women who received vaccine during pregnancy; women who receive MMR should not get pregnant within 1 month of vaccine administration.

\section{REFERENCES}

1. Rowson KE, Malik YBW. Human Papova (wart) virus. Bacterial RW 1967; 31: 110-31.

2. Zur Hausen H, deVilliers EM, Gissman L. Papillomavirus infections and human genital cancer. Gynecol Oncol 1981; 2(2 pt 2): 5124-8.

3. Bosch FX, Lorincz A, Munoz N, et al. The causal relation between human papillomaviruses and cervical cancer. J Clin Pathol 2002; 55: 244-65.

4. Trimble C, Hildesheim A, Brinton L, et al. Heterogeneous etiology of squamous cancer of the vulva. Obset Gynecol 1996; 87: 59-64.

5. deVilliers EM. Human Papillomaviruses-introduction. Semin Cancer Biol 1999; 9: 377.

6. Bryan JT, Brown DR. Transmission of human papillomavirus Type 11 infection by desquamated cornified cells. Virology 2001; 281: 35-42.

7. Evander M, Frazer IH, Payne E, et al. Identification of the alpha (6) integrin as a candidate receptor for papilloma viruses. J Virol 1997; 21: 2449-56.

8. Zur Hausen H. Papillomaviruses in human cancer. Proc AAP 1999; 111 : 581-7.

9. Brown DR, Schrader JM, Bryan JT, et al. Detection of multiple human papillomavirus types in condylomata acuminata lesions from otherwise healthy and immunosuppressed patients. J Clin Microbiol 1999; 37: 3316-22.

10. Hildesheim A, Han CL, Brinton LA, et al. Human papillomavirus type 16 and risk of pre-invasive and invasive vulvar cancer: results from a seroepidemiological case-control study. Obstet Gynecol 1997; 90: 748-54.

11. Bosch FX, Shiffman M, Solomon D, eds. Future directions in epidemiologic and preventive research on human papillomaviruses and cancer. J Nat'l Cancer Inst Mono. Vol. 31. Oxford, UK: Oxford University Press, 2003.

12. Dillner J, Von Krogh G, Horenblas S, et al. Etiology of squamous cell cancer of the penis. J Virol Nephrol Suppl 2000; 205: 189-93.

13. Smoltkins D. Virology of human papilloma virus. Clin Obstet Gynecol 1989; 32: 117-26.

14. Winer RL, Lee SK, Hughes JP, et al. Genital human papillomavirus infection: incidence and risk factors in a cohort of university students. Am J Epidemiol 2003; 157: 218-26.

15. Ho GY, Bierman R, Beardsley L, et al. Natural history of cervix vaginal papillomavirus infections in young women. N Engl J Med 1998; 338: 423-8.

16. Watts DH, Koutsky LA, Holmes KK, et al. Low risk of perinatal transmission of human papillomavirus: results from a prospective cohort study. Am J Obstet Gynecol 1998; 178: 365-73.

17. Mosher WD, Chandra A, Jones J. Sexual behavior and selected health measures: men and women 15-44 years of age. United States 2002. Adv Data 2006; 362: 1-55.

18. Franco EL, Villa LL, Sobrinho JP, et al. Epidemiology of acquisition and clearance of cervical human papillomavirus infection in women from a high-risk area for cervical cancer. J Infect Dis 1999; 180: 1415-23.

19. Molano M, Vanden BA, Plummer M, et al. Determinants of clearance of human papillomavirus infections in Colombian women with normal cytology: a population-based 5 year follow-up study. Am J Epidemiol 2003; 158: 486-94.

20. Weinstock H, Berman S, Cates W Jr. Sexually transmitted diseases among American youth: incidence and prevalence estimates 2000. Perspect Sex Reprod Health 2004; 36: 6-10.

21. American Cancer Society, Cancer Facts and Figures 2005. Atlanta, GA: ACS, 2005: 1-60.

22. Shiffman M, Soloman D. Findings to Date from the ASCUS-LSIL Triage study (ALTS). Arch Pathol LAB Med 2003; 127: 946-949.

23. Fleischer AB, Parrish CA, Glenn R, Feldman SR. Condylomata Acuminata (Genital Warts): Patient Demographics and Treating Physicians. Sex transmitted Dis 2001; 28: 643-647.

24. Myers ER, McCrory DC, Nanda K, et al. Mathematical model for the natural history of human papillomavirus infection and cervical carcinogenesis. Am J Epidemiol 2000; 157: 1158-71.

25. Revzina NV, Dielemento RJ. Prevalence and incidence of human papillomavirus infection in women in the USA: a systematic review. Int J STD AIDS 2005; 16: 528-37.

26. Burk RD, Kelly P, Feldman J, et al. Declining prevalence of cervicovaginal human papillomavirus infection with age is independent of other risk factors. Sex Trans Dis 1996; 23: 333-41.

27. Manhart LE, Holmes KK, Koutsky LA, et al. Human papillomavirus infection among sexually active young women in the United States: implications for developing a vaccine strategy. Sex Transm Dis 2006; 33: 502-8.

28. Wright TC Jr, Cox JT, Massad LS, et al. 2001 Consensus guidelines for the management of women with cervical cytological abnormalities. JAMA 2002; 287: 2120-9.

29. Reeves WC, Ruparelia SS, Swansen KI, et al. National registry for juvenile onset recurrent respiratory papillomatosis. Arch Otolaryngol Head Neck Surg 2003; 129: 976-82.

30. Armstrong LR, Preston EJ, Rechert M, et al. Incidence and prevalence of recurrent respiratory papillomatosis among children in Atlanta and Seattle. Clin Infect Dis 2000; 31: 107-9.

31. Davis BD, Dulbecco R, Eisen HN. Other Pox viruses that infect man. In: Hagerstown MD, ed. Microbiology. 2nd edn. Harper and Row, 1973: 1275.

32. Viac J, Churdonnet Y. Immuno competent cells and epithelial modifications in molluscum contagiosum. J Cutan Pathol 1990; 17: 202-5.

33. Cotton DWK, Cooper C, Barrett OF, et al. Severe atypical molluscan contagiosum infection in an immuno compromised host. Br J Dermatol 1987; 116: 871-6.

34. Thurn J. Human Parvovirus B19: historical and clinical review. Rev Infect Dis 1988; 10: 1005-11.

35. Botbart HA. Human parvovirus infections. Annu Rev Med 1990; 41: 25-34.

36. Rodis JF, Quinn DL, Gary WG, et al. Management and outcomes of pregnancies complicated by human B19 parvovirus infection: a prospective study. Am J Obstet Gynecol 1990; 163: 1168-71.

37. Torok TJ. Human Parvovirus B19 infections in pregnancy. Pediatr Infect Dis J 1990; 9: 770-6.

38. Humphrey W, Magoon M, O’Shaughnessey R. Severe nonimmune hydrops secondary to parvovirus B19 infection. Spontaneous reversal in utero and survival of a term infant. Obstet Gynecol 1991; 78: 900-2.

39. Modlin JF, Kinney JS. Perinatal enterovirus infections. Adv Pediatr Infect Dis 1987; 2: 57-78.

40. Cherry JD, Nelson DB. Enterovirus Infections: their epidemiology and pathogenesis. Clin Pediatr 1966; 5: 59-63.

41. Modlin JF. Perinatal echovirus and group B coxsackie virus infections. Clin Perinatal 1988; 15: 233-46.

42. Torfasion EG, Reimer CB, Keyserling HC. Subclass restriction of human enterovirus antibodies. J Clin Microbiol 1987; 8: 1376-9.

43. Rosenberg HS. Cardiovascular effects for congenital infections. Am J Cardiovasc Pathol 1987; 71: 147-56.

44. Korones SB. Uncommon virus infections of the mother, fetus and newborn: influenza, mumps and measles. Clin Perinatol 1988; 15: 259-72.

45. Sterner G, Grandien M. Mumps in pregnancy at term. Scand J Infet Dis 1990; 71: 36-8.

46. CDC. Mumps Prevention. Recommendations of the advisory committee on immunization practices (ACIP). MMWR 1991; 40: 1-91. 
47. CDC. Measles, mumps and rubella-vaccine use and strategies for elimination of measles, rubella and congenital rubella syndrome and control of mumps: recommendations of the advisory committee on immunization practices (ACIP). MMWR 1998; 47: RR-8.

48. Harling R, White JM, Ramsay ME, et al. The effectiveness of the mumps component of the MMR vaccine. A case control study. Vaccine 2005; 23: 4070-4.

49. CDC. Update: multistate outbreak of mumps-United States. January 1 May 2, 2006. MMWR 2006; 55: 1-5.

50. Notice to readers. Undated Recommendations of the advisory committee on immunization practices for the control and elimination of mumps. MMWR 2006; 55: 629-30.
51. Amstey MS. Measles in principles and practice of medical therapy in pregnancy. In: Gleicher NB, ed. 2nd edn. Stamford, CT: Appleton and Lange, 1998: 791-2.

52. Grandien M, Sterner G. Measles in pregnancy. Scand J Infect Dis 1990; 71: $45-8$.

53. Gazala E, Karplus M, Liberman JR, et al. The effect of maternal measles on the fetus. Pediatr Infect Dis 1985; 4: 203-4.

54. Horstmann DM. Rubella. Clin Obstet Gynecol 1982; 25: 585-98.

55. Freij BJ, South MA, Sever JL. Maternal rubella and the congenital rubella syndrome. Clin Perinatal 1988; 15: 247-57.

56. Munro ND, Sheppard S, Smithers RW, et al. Temporal relations between maternal rubella and congenital defects. Lancet 1987; 2: 201-4.

57. Gall SA. Rubella in pregnancy. Obstet Gynecol Rep 1990; 2: 161-9. 